\title{
Zero Renewable Incentive Analysis for Flexibility Study of a Grid
}

\author{
Peyman Mazidi ${ }^{1(\bowtie)}(\mathbb{D})$, Gregory N. Baltas ${ }^{1}$, Mojtaba Eliassi $^{1}$, \\ Pedro Rodriguez ${ }^{1,2}$, Ricardo Pastor ${ }^{3}$, Michalis Michael ${ }^{4}$, \\ Rogiros Tapakis ${ }^{4}$, Vasiliki Vita ${ }^{5}$, Elias Zafiropoulos ${ }^{5}$, \\ Christos Dikeakos ${ }^{6}$, and George Boultadakis ${ }^{7}$ \\ ${ }^{1}$ Loyola Institute of Science and Technology (LoyolaTech), \\ Loyola University Andalucia, Seville, Spain \\ pmazidi@uloyola.es \\ ${ }^{2}$ Research Center on Renewable Electrical Energy Systems (SEER), \\ Technical University of Catalonia Barcelona, Barcelona, Spain \\ 3 Centro de Investigação em Energia, REN - State Grid, S.A, Sacavém, Portugal \\ ${ }^{4}$ Transmission System Operator, Cyprus (TSOC), Strovolos, Cyprus \\ 5 Institute of Communications and Computer Systems, \\ 9 Iroon Polytechniou Street, 15780 Athens, Greece \\ ${ }^{6}$ Independent Power Transmission Operator (IPTO TSO), Athens, Greece \\ 7 European Dynamics Luxembourg SA, 12, Rue Jean Engling, \\ 1466 Luxembourg, Luxembourg
}

\begin{abstract}
Power systems with renewable sources are undergoing various changes to safely accommodate higher share of renewables. In this process, they require different changes ranging from technical characteristics to regulations. In order to manage technicality of the integration of the renewables, storage units play an important role. On the other hand, many countries allocate incentives to the green sources of energy. In this paper, we develop a flexibility-oriented dayahead market model that accounts for renewable sources and storage units where no incentive is provided to the renewable sources. Additionally, a flexibility indicator (FLEXIN) is adopted to demonstrate the provided flexibility to the system. FLEXIN is defined by accounting for five sources of flexibility, reserve from conventional power sources, available renewables, storage units, transmission line availability and active demand. The results demonstrate how a system can cope with renewable sources with no incentive in the presence of storage. This paper is part of the Horizon 2020 Flexitranstore project.
\end{abstract}

Keywords: Battery energy storage system $\cdot$ Flexibility $\cdot$ Power system

\section{Nomenclature}

\section{Indexes}

$i, j \quad$ Index of network buses

$h \quad$ Time interval (hour)

$g \quad$ Index of generating units 


\section{Sets}

$\Omega_{\ell}^{i} \quad$ Set of all buses connected to bus $i$

$\Omega_{T H}^{i} \quad$ Set of all thermal generating units connected to bus $i$

$\Omega_{\text {WIND }}^{i}$ Set of all wind generating units connected to bus $i$

$\Omega_{\text {BESS }}^{i} \quad$ Set of all BESS units connected to bus $i$

\section{Parameters}

$\alpha_{g} \quad$ Incremental production cost $\left(\$ / M W h^{2}\right)$

$b_{g} \quad$ Linear production cost $(\$ / \mathrm{MWh})$

$c_{g} \quad$ Fixed operation cost of generator $g(\$ / \mathrm{h})$

$r_{g} \quad$ Reserve cost of generator $g(\$ / \mathrm{h})$

VOLL Value of lost load (\$/MWh)

VOEMS Value of emissions $(\$ / \mathrm{Kg})$

$E M S_{g} \quad$ Emissions for generator $g(\mathrm{Kg} / \mathrm{MWh})$

$D_{h, i}^{a l} \quad$ Proposed active power demand in bus $i$ at time $h$ (MW)

$\zeta_{\min } / \zeta_{\max }$ Minimum and maximum active load variability $(\%)$

$P_{g}^{\max } \quad$ Maximum limit of active power generation of unit $g$ (MW)

$P_{g}^{\min } \quad$ Minimum limit of active power generation of unit $g$ (MW)

$\bar{R}_{g} \quad$ Ramp-up limit of generator $g(\mathrm{MW} / \mathrm{h})$

$\underline{R}_{g} \quad$ Ramp-down limit of generator $g(\mathrm{MW} / \mathrm{h})$

$\eta_{c} \quad$ Efficiency of charging (\%)

$\eta_{d} \quad$ Efficiency of discharging (\%)

$P_{i, j}^{\max } \quad$ Maximum active power flow of branch connecting bus $i$ to $j$

$P_{i, j}^{\min } \quad$ Minimum active power flow of branch connecting bus $i$ to $j$

$\gamma \quad$ Spinning reserve $(\%)$

$\rho_{g} \quad$ Startup cost of generator $g$ [\$]

$\sigma_{g} \quad$ Shutdown cost of generator $g[\$]$

$X_{i, j} \quad$ Reactance between lines $i$ and $j[\Omega]$

$\delta^{\max } \quad$ Maximum voltage angle

$\delta^{\text {min }} \quad$ Minimum voltage angle

\section{Variables}

$u_{h, g} \quad$ Commitment status of generator $g$ (1: on, 0:off)

$y_{h, g} \quad$ Startup decision of generator $g$ at time $h$ (1:up, 0:no change)

$z_{h, g} \quad$ Shutdown decision of generator $g$ at time $h$ (1:down, 0:no change)

$P_{h, g} \quad$ Active power generated by unit $g$ at time $h$ (MW)

$D_{h, i}^{d r P} \quad$ Realized demand in bus $i$ at time $h$ (MW)

$R_{h, g} \quad$ Reserve at time $h$ by generator $g$ (MW)

$P_{h, i, j} \quad$ Active power flow of branch connecting bus $i$ to $j$ at time $h$ (MW)

$D_{h, i}^{l s P} \quad$ Load shedding in bus $i$ at time $h(\mathrm{MW})$

$P_{h, g}^{c} \quad$ Active power charge into BESS $g$ at time $h$ (MW)

$P_{h, g}^{d} \quad$ Active power discharge from BESS $g$ at time $h$ (MW)

$\delta_{h, i} \quad$ Voltage angle of bus $i$ at time $h(\mathrm{rad})$

$S O C_{h, g} \quad$ State of charge of BESS $g$ at time $h$ 


\section{Introduction}

The current power system is still based on the past technologies where large-scale fossil fuel based power plants produce majority of the electricity share in the generation mix and consumers do not have significant participation [1]. Thus, the electricity market of the next decade will move towards a decentralized structure with more variable generation and improved technologies and increased consumer participation. On the other hand, in the balancing markets with centralized and decentralized (self-scheduling) structures, if there is a security threat to the system due to locational imbalances, the centralized market can solve such an issue where additional factors such as reserve and congestion are also simultaneously considered. Hence, there are still some European countries that consider a centralized system, such as Greece [2].

As renewables' production continues to increase in Europe, power systems and electricity markets face multiple challenges in order to successfully integrate large amounts of non-dispatchable renewable energy sources (RES). Figure 1 shows a perspective of the electricity generation outlook in the European Union (EU) per technology in a scenario that incorporates existing policies and announced policy intentions [3].

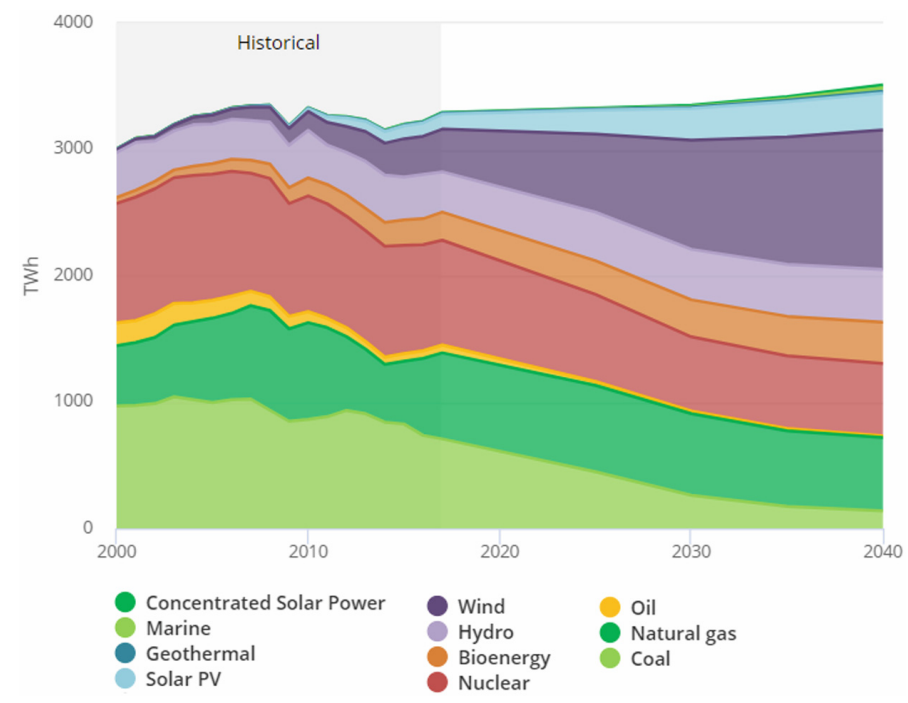

Fig. 1. Electricity generation outlook in the EU per technology. Source (IEA/WEO 2018) [3].

The political goals on the supporting mechanisms clearly state that renewable power plants must be integrated in the electricity market to create an open, competitive, transparent and non-discriminatory environment among all players [4]. Therefore, the economic sustainability of renewable generation in the electricity market is critical for pursuing the new energy policy in the EU. Without further incentives and with the end 
of the contracts for subsidized renewable production (e.g. feed-in-tariffs), renewable producers will be forced to participate under the same market rules as any other producer [5].

One key issue associated with RES is the stochastic nature of sources. High variability of RES requires an analogous degree of flexibility from the available resources to maintain power balance. At the same time, renewable producers are being challenged about how they can contribute to the system's flexibility. Advances in renewable generation technologies (e.g. smart maintenance [6]) are allowing them to provide more flexibility to the system. Thus, the provision of flexibility services is a key, both for renewable producers, as they can sell additional services and increase their revenue, and for the system operators, as they increase the list of flexibility sources that are available for the system's operation.

\section{Assumptions and Structure}

It is very common to consider that competitive markets without market failures can be modeled through an optimization programming where market surplus is maximized [7]. The model proposed in this paper for flexibility studies covers a day-ahead market operation as a social welfare maximization concept. In this regard, system operator makes the decisions in order to have the lowest operation cost in the next $24 \mathrm{~h}$ horizon. This is achieved by a mixed integer nonlinear programming problem (MINLP) where the objective is to minimize the operation cost of the system.

Renewables have incentives in many countries where they can receive subsidies for generation. This reduces the costs and assists them to provide low bids to the market. One other form of incentive is that renewable sources are prioritized over other types and this means that if there is any renewable energy available, they must be produced, regardless of the impact that this decision might have on the system, e.g. increase costs or create congestion. For this purpose, in this paper, specifically we do not consider any incentive for renewable sources. As a result, it is assumed that they participate in the market with their actual operating costs, which could still be lower than other participating generators.

\subsection{Reserve}

There are various methods to model reserve in a power system. Different operators consider different approaches based on their system requirements and perspectives [8]. In this paper, the secondary control reserve is defined as capability of committed generators to provide additional production in case it is required. On the other hand, the minimum reserve is considered as a percentage of system load at any time. Although it is assumed that the generator must have enough capacity to provide additional support, this excess support is not limited by the ramp characteristics of the generator. As a result, if a generator is already running and has free capacity, the free capacity is accounted for as the reserve. This simplification of neglecting the ramping limitation and solely considering committed capacity might result in solutions that are cheaper, easier to solve (due to mathematical difficulties) with unrealistic reserve values. 


\subsection{Flexibility Index}

Uncertainty, outage and variations can be considered as three main origins that raise the need for flexibility in a system. Uncertainty in the system can be defined as coming from various sources such as intermittent renewables. In a similar manner, outages of generators can significantly impact the system. Finally, variations in the demand could change the condition of the system. These parameters could show their influence in the ranges from real-time to medium term and long term. In other words, the system requires flexibility in control, operation and planning. In this paper, we try to address the flexibility from operation point of view, Fig. 2.

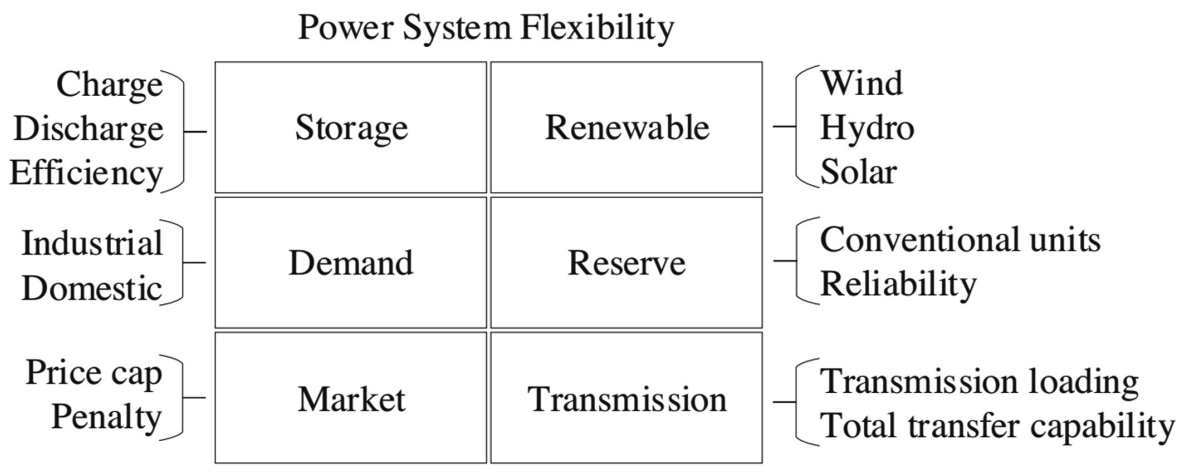

Fig. 2. Flexibility factors in a power system [9]

The three hierarchical levels that the flexibility can come into play are generation, transmission and distribution. Renewable sources and battery energy storage system (BESS) can produce at the generation level to provide flexibility. For the distribution, active load can provide flexibility by shifting the demand from one time to another. In the same way, a BESS could also play such a role in form of an active distribution node $(\mathrm{ADN})$. On the transmission level, loading of the transmission lines could be linked to the flexibility of the lines. For instance, if the lines in an area of the system are mostly fully loaded, the flexibility of the system from transmission point of view is very low. Since each transmission system operator is responsible for monitoring its grid, they calculate such concept as available transfer capability (ATC) [10]. Additionally, the ATC can be shared with the generation companies so that they can consider such flexibility in their operation planning as well. Considering these points, we also adopt the flexibility definition as a flexibility index (FLEXIN) provided by [9] to show the degree of system flexibility. 


\section{Modelling}

The proposed model is formulated through an optimization problem that is explained below. In the following, the case studies are defined and the input data of the test system are mentioned.

\subsection{Mathematical Formulation}

The mathematical formulations for the model have an MINLP form where the objective is to minimize operation cost of the whole system.

$$
\begin{aligned}
& \quad \sum_{h, g \in \Omega_{T H}^{i}, \Omega_{W I N D}^{i}}\left(\alpha_{g} P_{h, g}^{2}+b_{g} P_{h, g}\right) \\
& +\sum_{h, g \in \Omega_{B E S S}^{i}}\left(\alpha_{g} P_{h, g}^{c}{ }^{2}+b_{g} P_{h, g}^{c}+\alpha_{g} P_{h, g}^{d}{ }^{2}+b_{g} P_{h, g}^{d}+c_{g} S O C_{h, g}\right) \\
& +\sum_{h, g \in \Omega_{T H}^{i}}\left(r_{g} R_{h, g}\right)+\sum_{h, g \in \Omega_{T H}^{i}, \Omega_{W I N D}^{i}}\left(c_{g} u_{h, g}\right)+\sum_{h, g \in \Omega_{T H}^{i}, \Omega_{W I N D}^{i}}\left(\rho_{g} y_{h, g}\right) \\
& +\sum_{h, g \in \Omega_{T H}^{i}, \Omega_{W I N D}^{i}}\left(\sigma_{g} z_{h, g}\right)+\sum_{h, i}\left(V O L L \times D_{h, i}^{l s P}\right) \\
& +\sum_{h, g \in \Omega_{T H}^{i}} \operatorname{VOEMS} \times E M S_{g} \times P_{h, g}
\end{aligned}
$$

The operation cost of the system is presented in (1). It includes the production cost of conventional generators, wind generators and BESS in the first two terms. Cost of reserve is accounted for as well as commitment, startup and shutdown costs. Final two terms include the penalty for load shedding and cost of emissions. Subsequently, the system constraints are formulated as follows:

$$
\begin{array}{cc}
\sum_{g \in \Omega_{T H}^{i}, \Omega_{W I N D}^{i}} P_{h, g}+D_{h, i}^{l s P}-D_{h, i}^{d r P}-\sum_{g \in \Omega_{B E S S}^{i}} P_{h, g}^{c}+\sum_{g \in \Omega_{B E S S}^{i}} P_{h, g}^{d} \\
=\sum_{j \in \Omega_{\ell}^{i}} P_{h, i, j} & \forall h, i \\
u_{h, g} P_{g}^{\min } \leq P_{h, g} \leq u_{t, g} P_{g}^{\max } & \forall h, g \mid g \in \Omega_{T H, W I N D}^{i} \\
P_{h, g}-P_{h-1, g}-\bar{R}_{g} \leq 0 & \forall h, g \mid g \in \Omega_{T H}^{i}, \Omega_{W I N D}^{i} \\
P_{h-1, g}-P_{h, g}-\underline{R}_{g} \leq 0 & \forall h, g \mid g \in \Omega_{T H}^{i}, \Omega_{W I N D}^{i} \\
u_{h+1, g}-u_{h, g}=y_{h+1, g}-z_{h+1, g} & \forall h\left\langle N_{h}, g\right| g \in \Omega_{T H}^{i}, \Omega_{W I N D}^{i} \\
S O C_{h, g}=S O C_{h-1, g}+P_{h, g}^{c} \eta_{c}-\frac{P_{h, g}^{d}}{\eta_{d}} & \forall h, g \mid g \in \Omega_{B E S S}^{i}
\end{array}
$$




$$
\begin{aligned}
& P_{g}^{\min } \leq P_{h, g}^{c} \leq P_{g}^{\max } \\
& \forall h, g \mid g \in \Omega_{B E S S}^{i} \\
& P_{g}^{\min } \leq P_{h, g}^{d} \leq P_{g}^{\max } \\
& \forall h, g \mid g \in \Omega_{B E S S}^{i} \\
& P_{g}^{\min } \leq S O C_{h, g} \leq P_{g}^{\max } \\
& \forall h, g \mid g \in \Omega_{B E S S}^{i} \\
& S O C_{h, g}=0 \\
& \forall h=1, N_{h}, g \mid g \in \Omega_{B E S S}^{i} \\
& P_{h+1, g}^{c}-P_{g}^{\max }+S O C_{h, g} \leq 0 \\
& \forall h\left\langle N_{h}, g\right| g \in \Omega_{B E S S}^{i} \\
& P_{h+1, g}^{d}-S O C_{h, g} \leq 0 \\
& \forall h\left\langle N_{h}, g\right| g \in \Omega_{B E S S}^{i} \\
& P_{h, i, j}=\frac{\delta_{h, i}-\delta_{h, j}}{X_{i, j}} \\
& \forall h, i, j \mid j \in \Omega_{\ell}^{i} \\
& -P_{i, j}^{\max } \leq P_{h, i, j} \leq P_{i, j}^{\max } \\
& h, i, j \mid j \in \Omega_{\ell}^{i} \\
& \delta^{\min } \leq \delta_{h, i} \leq \delta^{\max } \\
& \forall h, i \\
& \delta_{h, i}=0 \\
& \forall h, i=\text { slack bus } \\
& \sum_{g \in \Omega_{T H}^{i}} R_{h, g} \leq \sum_{g \in \Omega_{T H}^{i}}\left(u_{h, g} P_{g}^{\max }-P_{h, g}\right) \\
& \forall h \\
& \sum_{g \in \Omega_{T H}^{i}} R_{h, g} \geq \gamma \sum_{i} D_{h, i}^{d r P} \\
& \forall h \\
& \sum_{h, i} D_{h, i}^{d r P}=\sum_{h, i} D_{h, i}^{a l} \\
& \left(1-\zeta_{\min }\right) \sum_{i} D_{h, i}^{a l} \leq \sum_{h, i} D_{h, i}^{d r P} \\
& \forall h \\
& \sum_{h, i} D_{h, i}^{d r P} \leq\left(1+\zeta_{\max }\right) \sum_{i} D_{h, i}^{a l} \\
& \forall h \\
& 0 \leq D_{h, i}^{l s P} \leq D_{h, i}^{d r P} \\
& \forall h, i
\end{aligned}
$$

Equation (2) shows the demand balance where transmission lines are considered. As a result, we obtain locational marginal prices in each node of the system as Lagrange multiplier of this equation.

Equation (3) limits minimum and maximum production level of conventional and wind generators. (4) and (5) set the upward and downward ramping capabilities of the generators. (6) provides the link between startup, shutdown and commitment status of the generators. 
Equations (7)-(13) are related to BESS. (7) shows the relation between charge, discharge and state-of-the-charge of the BESS by accounting for charge and discharge efficiencies. While (8)-(10) limit maximum and minimum charge, discharge and SOC values, (11) considers that the value of the SOC of the BESS should be the same at the beginning and the end of the simulation period. (12) considers that the amount that the BESS can be charged at any time should be less than the difference between the maximum capacity of the BESS and the SOC that is already available at the previous hour. Similarly, (13) considers that the amount of discharge of the BESS at any time cannot be more than the available amount of SOC at the previous time.

Equation (14) defines the power flow between the buses to be linearly related to the difference between the voltage angles of the buses, where (15) and (16) sets the limit on the maximum and minimum capacity of the lines and the voltage angles of the buses. (17) assumes that the voltage angle of the slack bus is zero.

Equation (18) considers that participation of each conventional generator in the hourly defined reserve in this system should be less than the maximum available capacity of that generator. The reserve should also be greater than some percentage of the realized load, (19).

Equations (20)-(23) consider the demand side management where moved sum of the demand in the simulation horizon should be equal to the sum of the forecasted demand. The demand flexibility degree is also considered as a percentage where the moved demand can only be a percentage of the forecasted demand, (21), (22). In the end, maximum load shedding at any time and bus should not be more than the realized $\operatorname{load}(23)$.

The mathematical formulation for FLEXIN is also provided in (24), where conventional generators provide flexibility through their ramping and reserve, BESS provides flexibility along with renewable sources individually, active demand from the customer side and ACT from transmission side also deliver flexibility.

$$
\begin{aligned}
& \sum_{g \in \Omega_{T H}^{i}} R_{h, g}+\sum_{g \in \Omega_{W I N D}^{i}}\left(P_{g}^{\max }-P_{h, g}\right)+\sum_{g \in \Omega_{B E S S}^{i}}\left(P_{g}^{\max }-S O C_{h, g}\right) \\
& +\sum_{i, j} \frac{\left(P_{i, j}^{\max }-\left|P_{h, i, j}\right|\right)}{2}+\zeta_{\max } \sum_{i} D_{h, i}^{a l}
\end{aligned}
$$

\subsection{Case Studies}

In order to investigate the performance of the model and analyze flexibility of the the system, different case studies have been defined and carried out, Table 1 . The main idea to design the case studies was to link the FLEXIN as a metric for evaluating flexibility of the system and demonstrating influence of various sources of flexibility. While Case 1 considers a basic case without the main flexibility sources, Case 5 puts together all the defined flexibility sources. 
Table 1. Considered study cases for the analyses

\begin{tabular}{l|l|l|l|l}
\hline Cases & Reserve & Renewable & Storage & Active demand \\
\hline Case1 & $\times$ & $\times$ & $\times$ & $\times$ \\
\hline Case2 & $\sqrt{ }$ & $\times$ & $\times$ & $\times$ \\
\hline Case3 & $\sqrt{ }$ & $\sqrt{ }$ & $\times$ & $\times$ \\
\hline Case4 & $\sqrt{ }$ & $\sqrt{ }$ & $\sqrt{ }$ & $\times$ \\
\hline Case5 & $\sqrt{ }$ & $\sqrt{ }$ & $\sqrt{ }$ & $\sqrt{ }$ \\
\hline
\end{tabular}

The model is coded in GAMS software v25.0.3 [11] and solved with BARON solver v17.10.16 [12] on a computer with 64 GB of RAM and i7-7820X @3.60 GHz Intel processor. As for the test system, we considered modified IEEE 24-bus test system [13].

\subsection{Input Data}

Penalty for performing a load shedding is considered by defining the value of lost load (VOLL) parameter as 10000 (\$/MWh). The value of emissions (VOEMS) for conventional generators is considered to have a cost of $20(\$ / \mathrm{kg})$. Charge and discharge efficiencies of BESS are assumed to be $98 \% .0 .9$ and 1.1 in radian are the minimum and maximum allowable values for voltage angles at each bus. The reserve has a value of $20 \%$ with respect to the demand $(\gamma)$, which means the system reserve at any time should be more than or equal to the $20 \%$ of the system demand. Finally, demand flexibility at each bus assumed to be $4 \%$ ( $\zeta)$. The rest of the data about the test system can be found in [9]. The model considers three BESS units, two wind plants and twelve nonrenewable sources.

It should be reminded that the wind generators are connected to the grid in buses 5 and 9 and the three BESS units are connected to the grid in buses 10, 13 and 18. The value of demand at hour 20:00 in bus 5, 9, 10, 13 and 18 is 70, 124, 146, 212 and $242 \mathrm{MW}$, respectively. The connecting transmission lines for the renewable generator buses are: L3 (b1-b5), L6 (b3-b9), L8 (b4-b9), L9 (b5-b10), L12 (b8-b9), L14 (b9b11), L15 (b9-b12). Similarly, the connecting transmission lines for the BESS buses are: L9 (b5-b10), L10 (b6-b10), L13 (b8-b10), L16 (b0-b11), L17 (b10-b12), L18 (b11-b13), L20 (b12-b13), L22 (b13-b23), L29 (b17-b18) and L31 (b18-b21).

\section{Results}

A comparison between the maximum and minimum changes of prices and loading of the lines are provided in Table 2. As it can be seen, addition of reserve in Case 2 has resulted in similar prices as in Case1, where no reserve is accounted for. Two explanations can be provided here. The first explains that in Case1, the system is already providing enough reserve, which by explicitly considering the $20 \%$ reserve, the operation cost of the system does not change significantly. 
Table 2. Price and line loading summary

\begin{tabular}{l|l|l|r|l}
\hline \multirow{2}{*}{ Cases } & \multicolumn{2}{l|}{ Price (\$/MWh) } & \multicolumn{2}{l}{$\begin{array}{l}\text { Transmission } \\
\text { loading (MW) }\end{array}$} \\
\cline { 2 - 5 } & Max & Min & Max & Min \\
\hline Case1 & 31.24 & 28.18 & 1048.74 & 508.99 \\
\hline Case2 & 31.20 & 27.64 & 1253.20 & 535.29 \\
\hline Case3 & 29.66 & 27.20 & 1026.62 & 563.25 \\
\hline Case4 & 30.88 & 27.25 & 942.30 & 456.63 \\
\hline Case5 & 30.62 & 27.22 & 1209.39 & 650.41 \\
\hline
\end{tabular}

The other explanation is that this type of formulating reserve, (18) and (19), does not accurately display the impact of reserve on the operation cost of the system. This is also in line with the previous explanation as this kind of formulation neglects the characteristics of the generators from ramping perspectives. Therefore, it can be concluded that the reserve formulation in [9] provides a more realistic model for reserve. However, as mentioned earlier, the current formulation has simpler form as it avoids the Big-M formulation. The impact of this formulation on the rest of the case studies should be taken into account when analysing the results.

Another observation from Table 2 is the distinct impact of renewables on the prices in Case3. One interesting reflection is the reduction in loading of the lines when storage enters the operation in Case4. Both maximum and minimum loading values have been reduced, where similar prices as in Case 3 are obtained. This shows how adding storage can relieve some stress from transmission lines in overall. Finally, by bringing in demand flexibility, although the prices see a reduction, the loading of the lines increases. It should be mentioned that there is no cost for loading of the lines in the operation cost.

Figure 3 illustrates different loading levels of the transmission lines at peak hour, 20:00 with the value of 2233 MW. It should be mentioned that in Case1, none of the flexibility sources are considered and Case 5 considers that only four of the flexibility sources have direct impact on the operation cost. The only flexibility factor that is not considered in the operation cost is the loading of the lines. Thus, it is not expected that the loading of the lines are minimized.

Comparing the two cases in Fig. 3 and considering the values in Table 2, it can be seen that adding the sources of flexibility to the system causes a decrease in the operation cost and an increase in the loading of the transmission system. Furthermore, it can be seen that except for L8 and L12, the loading of the mentioned lines increases by considering new flexibility sources.

Moreover, it can also be seen that except for L9, L13 and L22, the loading of the lines have reduced. As a result, in short, out of sixteen related lines to the flexibility sources, nine lines have had decrease in their loading values after consideration of flexibility sources. It should however be mentioned that the overall loading of the transmission system has increased. Thus, the flexibility sources have provided some loading relief (congestion management) for the connected buses at the peak hour. 


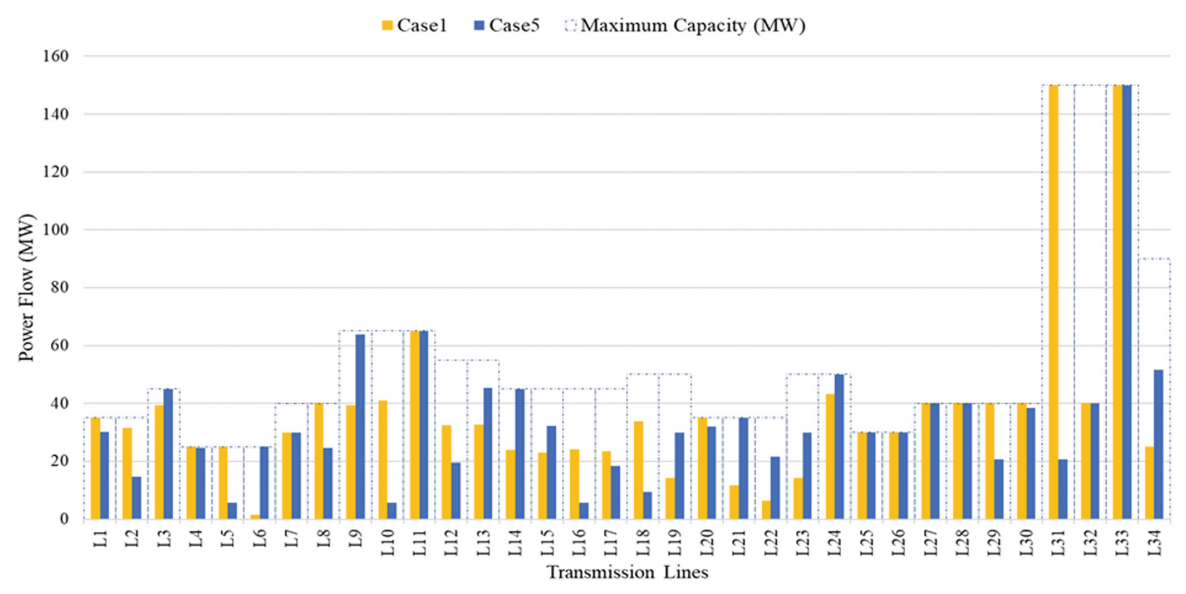

Fig. 3. Transmission line loading for Case1 and Case5.

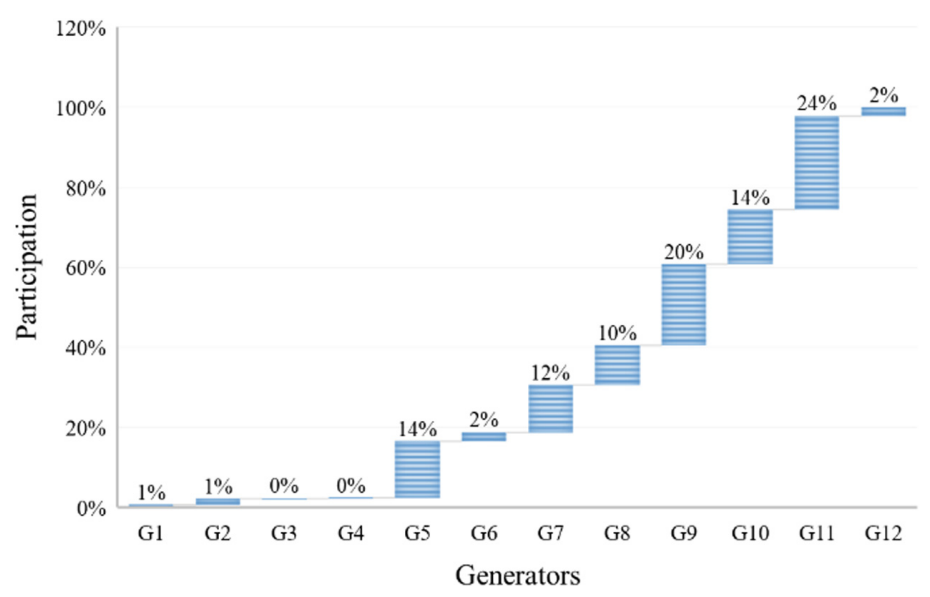

Fig. 4. Production share in Case2.

Figure 4 illustrates the generation percentage from generators, with G11 having the highest value. G11 is a conventional generator with maximum capacity of $440 \mathrm{MW}$. G11, G9 and G5 are the largest generators having about $60 \%$ of the production share. The $0 \%$ for $\mathrm{G} 3$ and $\mathrm{G} 4$ is due to rounding of the values.

Figure 5 displays the operation of batch of BESS in the system where all sources of flexibility are considered. It can be seen that the BESS starts charging when there is large amount of renewables and discharge in high load hours (h9, h10) and near peak and peak hours (h13, h14, h20, h21) (arbitrage condition). It is also interesting to see that the BESS is out of operation solely for four hours in the whole day. 


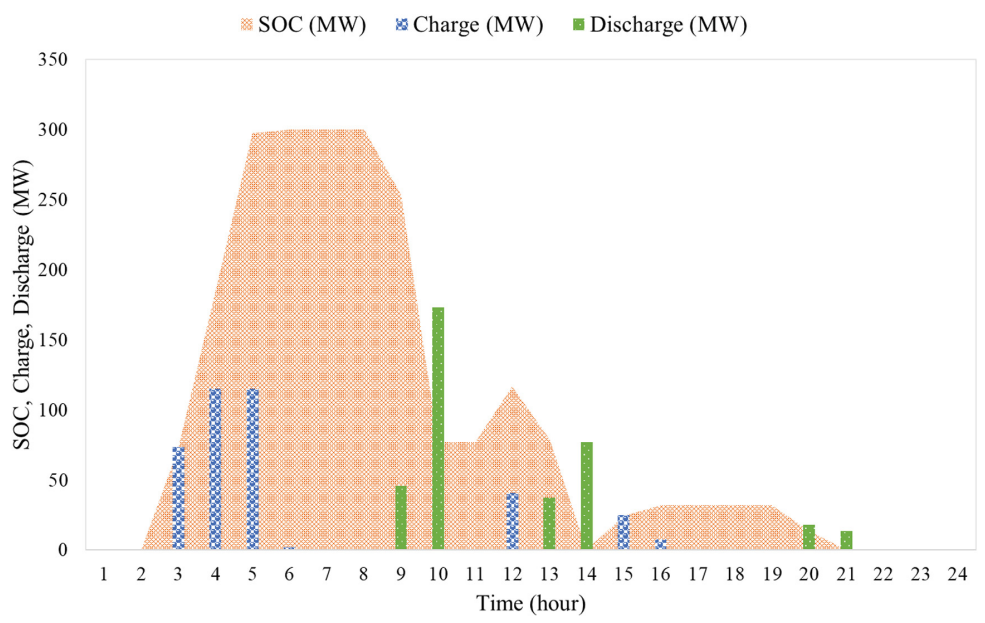

Fig. 5. Operation of aggregated BESS in Case5.

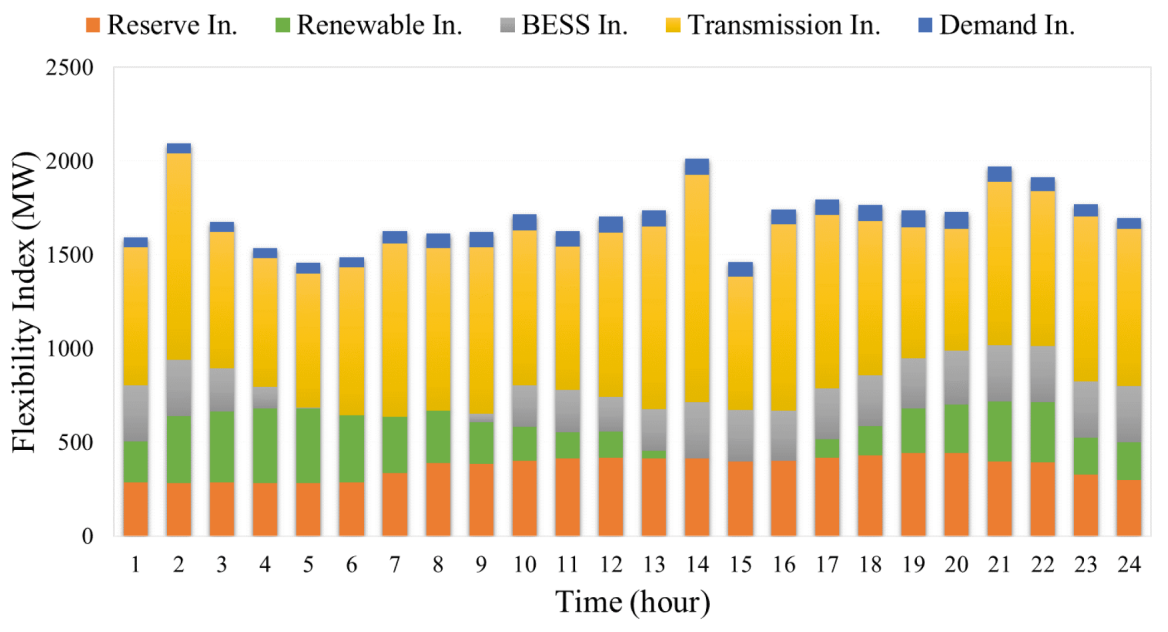

Fig. 6. FLEXIN for Case5.

Finally, Fig. 6 illustrates the hourly system flexibility provided from the considered five flexibility sources. It can be seen that on this particular day, the maximum and minimum system flexibility are calculated to be $2096 \mathrm{MW}$ (at hour2) and $1456 \mathrm{MW}$ (at hour5). The lowest value of flexibility occurs at hour5 resulting from three consecutive charging periods by the BESS. Now, this can be considered as a reference study for the operator. For instance, the operator can later look back, check the operating conditions on this day, and see whether they were satisfactory. If not, the operator can modify the system in order to achieve better system flexibility and security. Considering that there 
are various parameters affecting the flexibility, the operator could particularly ask BESS to be in standby at hour5 in case there is a contingency.

\section{Conclusions}

This paper incorporates five sources of flexibility into a day-ahead operation in power systems where the incentive for renewable sources are ignored. The paper also considers a simplified formulation for the reserve and the results show the impact of such simplification on the operation cost and flexibility. Finally, the adopted flexibility index displays how a system operator could investigate flexibility of the grid over a future operating horizon and make required adjustments to improve the operation and hedge against contingencies. Future studies could discuss the sensitivity of change in the operation cost and flexibility of the system with respect to various values of incentive for renewable sources. Additionally, self-scheduling aspect could also be adapted in order to demonstrate the possible modifications in case the decentralized approach is selected.

Acknowledgments. This work was supported by the European Commission under project FLEXITRANSTORE-H2020-LCE-2016-2017-SGS-774407 [14] and by the Spanish Ministry of Science under project ENE2017-88889-C2-1-R. Any opinions, findings and conclusions or recommendations expressed in this material are those of the authors and do not necessarily reflect those of the host institutions or funders.

\section{References}

1. Proposal for a regulation of the European parliament and the council on the internal market for electricity. European Commission, Brussels (2017)

2. Supporting document for the network code on electricity balancing: European network of transmission system operators for electricity (ENTSOE), Brussels (2014)

3. IEA/World Energy Council: World Energy Outlook 2018 (2018). https://www.iea.org/weo/

4. European Commission: COMMUNICATION FROM THE COMMISSION - Clean Energy for All Europeans, 30 November 2016. https://eur-lex.europa.eu/legal-content/EN/TXT/? qid=1481278671064\&uri=CELEX:52016DC0860

5. Imperial College London, Nera Economic Consulting, DNV GL: Integration of renewable energy in Europe. Final report for the European Commission (2014)

6. Mazidi, P.: From condition monitoring to maintenance management in electric power system generation with focus on wind turbines, KTH Royal Institute of Technology, Comillas Pontifical University, Delft University of Technology, p. 222 (2018)

7. Kazempour, J., Hobbs, B.F.: Value of flexible resources, virtual bidding, and self-scheduling in two-settlement electricity markets with wind generation-part I: principles and competitive model. IEEE Trans. Pow. Syst. 33(1), 749-759 (2018)

8. Ela, E., Milligan, M., Kirby, B.: Operating reserves and variable generation. National Renewable Energy Laboratory (NREL) (2011)

9. Mazidi, P., Baltas, G.N., Eliassi, M., Rodriguez, P.: A model for flexibility analysis of RESS with electric energy storage and reserve. In: 7th International Conference on Renewable Energy Research and Applications (ICRERA), Paris (2018) 
10. Christie, R.D., Wollenberg, B.F., Wangensteen, I.: Transmission management in the deregulated environment. Proc. IEEE 88(2), 170-195 (2000)

11. GAMS Development Corporation: General Algebraic Modeling System (GAMS), Washington, DC, USA (2018)

12. Tawarmalani, M., Sahinidis, N.V.: A polyhedral branch-and-cut approach to global optimization. Math. Program. 103(2), 225-249 (2005)

13. IEEE Commmitte Report: IEEE reliability test system. IEEE Trans. Power Apparatus Syst. PAS-98(6), 2047-2054 (1979)

14. FLEXITRANSTORE: An Integrated Platform for Increased FLEXIbility in smart TRANSmission grids with STORage Entities and large penetration of Renewable Energy Sources, European Union's Horizon 2020 research and innovation programme 2017-2021

Open Access This chapter is licensed under the terms of the Creative Commons Attribution 4.0 International License (http://creativecommons.org/licenses/by/4.0/), which permits use, sharing, adaptation, distribution and reproduction in any medium or format, as long as you give appropriate credit to the original author(s) and the source, provide a link to the Creative Commons license and indicate if changes were made.

The images or other third party material in this chapter are included in the chapter's Creative Commons license, unless indicated otherwise in a credit line to the material. If material is not included in the chapter's Creative Commons license and your intended use is not permitted by statutory regulation or exceeds the permitted use, you will need to obtain permission directly from the copyright holder.

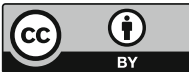

